\title{
An Ethnographic Study Exploring Football Sessions for Medium-Secure Mental Health Service-Users: Utilising the CHIME Conceptual Framework as an Evaluative Tool
}

\author{
Adam Benkwitz $\cdot$ Mervyn Morris $\cdot$ Laura C. Healy $\mathbb{D}$
}

Received: 6 January 2019/ Accepted: 11 February 2019/Published online: 19 February 2019

(C) The Author(s) 2019

\begin{abstract}
A key part of developing an understanding of 'what works' within the evolving mental health recovery evidence base is finding ways of serviceusers (and their friends and family) and practitioners working collaboratively. This interaction is slowly shifting practice, whereby care is potentially coconstructed in a setting between those involved to facilitate recovery-oriented processes. Increasingly, mental health services are appreciating the potential role of sport. This study adds to this body of literature by providing analysis of a football project in a medium-secure service context. This study also expands the methodological and theoretical scope of the literature by adopting an ethnographic approach and by utilising the CHIME conceptual framework as an evaluative tool. 47 participants were involved in the study, which included service-users, staff and volunteers. The data demonstrated that these sessions have
\end{abstract}

\section{A. Benkwitz}

Department of Health and Behavioural Sciences, Newman University, Birmingham, UK

M. Morris

School of Health Sciences, Birmingham City University, Birmingham, UK

L. C. Healy ( $\square)$

Sport, Health and Performance Enhancement (SHAPE)

Research Group, Department of Sport Science, School of

Science and Technology, Nottingham Trent University,

Clifton Lane, Nottingham NG11 8NS, UK

e-mail: laura.healy@ntu.ac.uk considerable links to the CHIME processes, and can therefore be considered to enhance personal recovery for those involved.

Keywords Recovery - CHIME - Ethnography · Mental health $\cdot$ Service-users

\section{Recovery Context}

The meaning of 'recovery' in the context of mental health is evolving [1, 2], slowly, away from the traditional notion of 'clinical recovery', i.e., an outcome observed by an expert [3], to appreciate the importance of lived experience [4] in an ongoing, personal journey [5] of 'personal recovery', i.e., a subjectively viewed and valued process [6, 7]. This acceptance that each individual's experience is different suggests that there is no blueprint for recovery [8], however, drawing on the literature pertaining to service user's accounts, there are key facets of recovery that have been systematically identified by Leamy et al. [9] as part of the broader REFOCUS research programme on recovery. Leamy et al. [9] conducted a systematic review and narrative synthesis that provided an empirically based conceptual framework of personal recovery in mental health. 97 studies were utilised in order to identify five recovery processes that are important to recovery. These 
processes were articulated by the acronym CHIME, which comprised of: connectedness (including peer support, support groups, relationships, support from others, being part of the community), hope and optimism (which was having belief in the possibility of recovery, motivation to change, hope-inspiring relationships, positive thinking and valuing success, and having dreams and aspirations), identity (that involved dimensions of identity, rebuilding a positive sense of identity, and overcoming stigma), meaning in life (that involved the meaning of mental illness experiences, spirituality, quality of life, meaningful life and social roles and goals, and rebuilding life), and empowerment (that encompassed personal responsibility, control over life, and focusing upon strengths). The criticism of personal recovery is that it can be deemed 'complex and disordered' [10], therefore the robust development of this conceptual framework is useful to bring some order to the exploration and evaluation of mental health recovery focused practice and services [9].

A key part of developing an understanding of "what works' within the evolving recovery evidence base is finding ways of service-users (and their friends and family) and practitioners working collaboratively [11]. Arguably, this interaction is (slowly) shifting practice, whereby care is potentially co-constructed in a setting between those involved in order to facilitate recovery-oriented processes [12]. There is an increase in studies in mental health appreciating the experiences of service-users and staff (and others) [13, 14]. However, researchers developing the evidence base for these types of practice need to remain mindful of the power relations still present [15], for instance, staff researchers who undertake research with service-users under their care. Being mindful of this caveat, and as discussed further in subsequent sections, the current study gathered data in situ from service-users, volunteers and staff members, and was undertaken by a researcher who was not a service-user, a volunteer or a staff member, in order to explore differing perspectives (and potential power relations) within this specific sporting context. Increasingly, mental health services are appreciating the potential role of sport, physical activity and/or exercise [16].
Mental Health and Sport, Physical Activity and/ or Exercise

Despite some notable contributions, literature on the potential for sport and physical activity to contribute to recovery in a positive sense remains scarce, and often methodologically flawed or vague [17]. Furthermore, as Carless and Douglas [16, p 140] stated "research has tended to focus on the ways exercise may alleviate symptoms, impairment, and dysfunction rather than its potential to contribute meaning, purpose, success, and satisfaction to a person's life", or in other words, there remains a dominance of the simplistic dose-response relationship of certain specific exercises [e.g., see $18,19]$ due to the assumed authority [20] of the 'clinical gaze' within both health services and sport science. Often these pre- and post-intervention measures 'explain' positives via psychological [e.g., selfefficacy, distraction, self-esteem, see 21, 22] or physiological/biological explanations [e.g., see 23], which increases the biomedical model focus at the expense of valuing and learning from the varied lived experiences of, and providing a voice for, the people actually involved. This is despite the Department of Health's [24] 'Future in Mind' policy specifically highlighting the scope available for general practitioners and other professionals to offer social prescribing of activities such as sport (but does not mention exercise or physical activity) to improve wellbeing and mental health in children and young people. Similarly, as Smith et al. [25] discussed, the Government's 'Sporting Future' strategy places emphasis on mental wellbeing within the nation's sporting agenda, with the 'measure' of the link between these elements being "improved subjective wellbeing”. [26, p 74] This limited, but potentially significant, change in policy rhetoric highlights a move away from the dominance of the clinical gaze towards listening to and valuing people's experiences, which could be argued to be in line with the slow but steady evolution from clinical towards personal recovery $[2,7]$. However, the current evidence base in this area is "more complex and nuanced than is perhaps commonly assumed, policy-makers and practitioners face a number of challenges in seeking to provide government with evidence of the contribution made by sport participation". [25, p 11] An example of the complexity could include the differences between an individual undertaking an exercise at a specific 
intensity but on their own (for example, long distance running), as opposed to team sports that might involve twenty or thirty people interacting together in one place but with varying levels of movement and intensity, for example, a goalkeeper in football will move far less than an outfield player, but they may benefit in many other ways due to the social nature of sport, as opposed to the potentially isolating types of exercise and physical activity that could be undertaken. Equally as important could be the context of the activity (health service or community based), how activities are run, by whom and for whom, and whether they have an underpinning focus on competition, recreation or health. One such area that is developing a participant-focused evidence base to explore this complexity is football for mental health projects.

\section{Football Specific Projects}

There has been an increase in the use of football (in various formats) to aid recovery, most often in partnership with football clubs [27], however, literature on initiatives that are located and run solely by mental health services is limited. Furthermore, research that centres on medium-secure service-users and staff is rarer still. Rather than a distracting predominance on dose-effect style studies, the nature of a team sport like football brings the social interaction and group dynamics to the fore [28]. Therefore, existing studies have highlighted the importance of moving away from exercising for periods of time at certain intensities and instead raising issues like: football being a site and topic to break down barriers, for example, talking to new people or opening up about health concerns [29-31], football projects tackling stigma [4], or helping people to (re)discover their identity [17] and recover personal and social roles [32].

Qualitative literature has also focused on the beneficial function of football to initially engage with people, then to have something to talk about (football), which builds rapport and enables participants to discuss issues and challenges [33]. This is especially important to engage 'hard to reach' populations within a mental health context. Research suggests that men's reluctance to seek support can further exacerbate distress and can often lead to suicide [34], and initial studies have suggested that football can be useful to engage these often 'hard to reach' male demographics who are most at risk [35, 36].

\section{Summary of Literature and Rationale for Study}

Whilst the existing literature is a solid starting point, it could be argued that further development of this evidence base is required, with consideration given to four areas specifically. Firstly, the context within which the project is delivered is potentially important, as it should not be assumed that projects that are delivered in the community by football clubs are synonymous with projects delivered within a therapeutic, mental health worker delivered context [e.g., the 'It's a Goal' project, 31, 37], or considered to be identical to a project that involved qualified football coaches and then a therapeutic programme running alongside [4]. Secondly, Magee et al. [4] were critical of the projects they studied as they retained a heavy biomedical model approach, which is often part of time-specific projects that inevitably become affixed with targets and outcome measures. Mental health services could take note of this small but significant idiosyncrasy, and scholars should be mindful of the underpinning aims (and therefore, potentially 'outcome measures') and the sustainability of sport projects, for instance, considering whether they are genuinely recovery focused. Thirdly, from the information available, methodological approaches in this area appear to be limited to generic questionnaire data and/or interviews, there is a lack of researcher involvement actually at the sessions (participant observation), which could be a key omission if the aim is to explore what goes on at projects. Finally, the existing literature often lacks a coherent theoretical or conceptual approach to develop analysis and inform future work.

Therefore, addressing those four points, this project aimed to add to this body of literature by (a) providing analysis of a sporting project that aimed to improve mental health within a certain context, which (b) adds to the qualitative data exploring lived experiences. This study also contributes by (c) expanding the methodological and theoretical scope of the literature by adopting an ethnographic approach and by (d) adopting the CHIME conceptual framework, which aims to draw together the recovery-focused literature and the relevant sport-specific studies. 


\section{Methodology}

In terms of the broader research context for this study, the emergent priority is the development and evaluation of interventions to support the five CHIME recovery processes $[9,11]$. It therefore follows that if recovery is subjective and best judged by the person living with the experience [3], then initiatives should be evaluated by exploring and providing a 'partial interpretation' [38] of those lived experiences. This approach aligns with the underpinning philosophical assumptions of this study, of a relativist ontology (assumes numerous subjective realities) and a constructionist epistemology (our understanding is based on appreciating multiple social constructions of knowledge) [39]. Therefore, this study adopted a qualitative approach to explore what it is like [40] to experience the football sessions for those involved (service-users, staff and volunteers). This study has gained unique access to an ongoing NHS Mental Health Foundation Trust football project, which has been running for several years (as opposed to being a specific, finite project). Therefore, this can be considered a naturalistic study that seeks to explore and further understand the relationship between football and mental health for those involved (service-users, staff and volunteers) in order to inform policy and practice.

\section{The Football Sessions}

Sessions run each Tuesday morning for $90 \mathrm{~min}$, in a well-equipped indoor football arena in the centre of a large city, and are run by an occupational therapy team based within a medium-secure unit. The sessions are attended by service-users from numerous mediumsecure units across the city, who have been authorised to be chaperoned by members of Trust staff in order to travel to the venue and partake in small sided games of football. There are two features of the sessions to note, firstly, there are also service-users and former serviceusers who attend who have transitioned out of the medium-secure setting and are engaged with accommodation services or who are back in the community (and still engaging with services). Secondly, several members of Trust staff, and also volunteers, play football as part of the sessions alongside service-users, all of whom were eligible to be part of the study as they had lived experience of the sessions. Each week there are between 40 and 50 people present at the sessions, with usually between 30 and 40 people who play football, as some staff members (chaperones) do not play and there are also some service-users who attend but for various reasons do not play, instead there are seating areas next to the pitch for them to spectate.

\section{Research Design}

A research design was required that was flexible enough to engage with the complexity of the context, given the involvement of varied demographics, given diagnoses, periods of time engaged with various services, as well as the involvement of staff and volunteers, which are challenges that have been highlighted within sport and mental health settings previously [16, 41]. There were also the expectations to inform future practice of social inclusive, nonstigmatising activities [42] that aid recovery and provide a voice to participants [43]. This inclusivity began at the start of the research project via 'coproduction', as participants (service-users, staff and volunteers) were involved in discussions regarding the nature of the research. Including participants was both beneficial to the quality of the study and also a stipulation for gaining full NHS ethical clearance, which was gained in addition to the lead researcher's institutional ethical clearance. Central for the participants was the need not only for the researcher to interview them (provide a voice), but also to attend the sessions regularly to see what they entail. These methods, along with the underpinning philosophical assumptions and the need for flexibility to explore the complexity led to an ethnographic research design being adopted, which included participant observation and semi-structured interviews.

\section{Procedure}

As the sessions were already established, and as the study aimed to explore the lived experiences of everyone involved (service-users, staff and volunteers), the recruitment process began by briefing those present at the sessions on the study, giving them participant information sheets and initially asking them to consider being part of the participant observation data collection. As attendance fluctuated, and in order to attempt to inform everyone who attended prior to data collection beginning, this briefing period 
lasted for 3 weeks. During this period, participant information sheets were passed electronically to staff members who were known to accompany serviceusers to sessions but who were not present at the football sessions for the 3 weeks during the briefing period. Trust staff were also asked to pass information to anyone due to start attending sessions for the first time during the data collection period, and the researcher's contact details were provided to facilitate any discussions that were required. Those individuals that did not consent to be part of the participant observation were informed that they could continue to attend the sessions as normal, and that the researcher would not collect data (i.e., take any field notes) that related to them in any way. Once all reasonable steps had been taken to inform attendees about the study and written informed consent was gained from participants willing to take part in the study, the researcher attended the sessions as a participant-as-observer [44], in the sense that the researcher attended sessions, played football, sat and watched others play, and had informal conversations with other attendees, but was known to be a researcher (i.e., the researcher was participating in activities but not researching 'covertly'). After several months of participant observation, and once initial themes began to emerge, participants were purposively sampled [45] for semistructured interviews in order to explore emergent themes in more depth, with a new participant information sheet and informed consent form being signed.

\section{Participants}

There were 47 participants who consented for the participant observation data collection (36 serviceusers/former service-users, nine Trust staff members, two volunteers), which lasted for 46 weeks. There were seventeen semi-structured interviews undertaken (ten service-users, five staff and two volunteers). The study did not seek to access any medical or case files, and did not ask staff about their specific roles. This was due to the study having an inductive approach that focused on the experiences of those attending without the potential distractions of the 'clinical setting', which is in line with a personal recovery philosophy [9]. As with other football for mental health projects, the majority of participants were male (three were female), as football remains a contested site where gender relations continue to be reproduced, maintained and resisted [46]. More broadly, in this context, this could be partially viewed as a positive, as football projects can attract 'hard to reach' men who are known to under-use health services [36, 47, 48].

\section{Findings}

Participant observation data (from 47 participants) and interview data (from seventeen participants) were initially analysed thematically [45] through an inductive process, with first order themes identified and subthemes developed through an interplay between data and theory throughout the ethnographic process [49]. For this article, and in a similar manner to Brijnath [50], an additional step was taken to code data in line with the CHIME framework [9] in a deductive process, which meant that the data coded under one code name were categorised into two or three subcomponents within the overall analysis. This was done in order to both evaluate the football sessions against the well-established and evidence-based CHIME framework and to also locate the current study within the broader recovery literature. Table 1 provides an overview of the key themes from the initial inductive analysis, all of which were apparent throughout both the participant observation and interview data, before the discussion section focuses in more depth on the deductive analysis in order to explore the CHIME framework processes within this specific context.

\section{Discussion}

It is useful when considering the discussion of the CHIME processes to be mindful of the extent that service-users in medium-secure units, and those transitioning back into the community, might experience in their daily lives the opposite of these processes, in other words, feeling isolated (rather than having connectedness), feeling hopeless (rather than hopeful), lacking a sense of identity (rather than retaining a sense of their identity), and so forth. The following sections are based on the CHIME framework [9] and are structured in order of importance and relevance based on the interpretation of data in this study. 
Table 1 First and second order themes from inductive thematic analysis

\begin{tabular}{|c|c|}
\hline First order category & Second order category \\
\hline 1. Benefitting mental and physical health & $\begin{array}{l}\text { 1.1 Feel more positive after playing } \\
\text { 1.2 Feel physically fitter after playing, want to do more outside of sessions } \\
\text { 1.3 Physical and mental health noticeably deteriorate after missing numerous } \\
\text { sessions } \\
\text { 1.4 Looking forward to sessions and playing more }\end{array}$ \\
\hline $\begin{array}{l}\text { 2. Power relations between service-users, staff } \\
\text { and volunteers }\end{array}$ & $\begin{array}{l}\text { 2.1 Making decisions and feeling empowered } \\
\text { 2.2 Appreciating different/more human side to people ('Breaks down barriers') } \\
\text { 2.3 Staff felt football sessions aided therapeutic relationships with service-users } \\
\text { 2.4 'We're all the same out on the pitch' } \\
\text { 2.5 Safe social space, friendly, no arguments }\end{array}$ \\
\hline 3. Opportunity to play/be active & $\begin{array}{l}\text { 3.1 Lack of other opportunities to be active in their lives } \\
\text { 3.2 Recover former identity/social role ('I used to be a footballer before I became } \\
\text { ill') } \\
\text { 3.3 Desire for more sessions and more opportunities } \\
\text { 3.4 Anxiety about sessions ending ('Limited funding') } \\
\text { 3.5 Joining a team in the future } \\
\text { 3.6 Enjoying doing something meaningful }\end{array}$ \\
\hline 4. Shared/collective experiences & $\begin{array}{l}\text { 4.1 Felt part of something ('It's like a family here') } \\
\text { 4.2 Making new connections/social networks } \\
\text { 4.3 Highlighted similarities amongst the group } \\
\text { 4.4 Collective space over time ('People might leave for a while, then come back } \\
\text { and they're welcomed') } \\
\text { 4.5 Football is important, we all love football }\end{array}$ \\
\hline
\end{tabular}

\section{Connectedness}

The participants frequently discussed the importance of the social elements of the sessions, as they facilitated interactions (and friendships) that otherwise would not occur. This was especially significant for those from medium-secure units, as service-users highlighted that they would probably not have got out of bed if they did not have the sessions to attend, and for some it was the only time in the week that they left the unit. It was evident from the participant observation that there was a strong element of community and connectedness amongst participants, with a welcoming and friendly culture that was very much valued, as Marty (Volunteer) suggested:

I've been here for a very long time, I've seen the change in people. They've made a lot of friends. They feel here they can get involved, where years ago they were very quiet, shy. They involve their self with other people, talk and communicate. Look at 'Gerald' for example, when he first came, he was very quiet. He never got involved. He couldn't even touch a ball. You look at him now and he's fantastic. He's cheerful. He's happy. When you see him, he shakes your hand and he gets on with people. It wouldn't have happened if he couldn't come here and see everyone.

This supports previous studies, highlighting the social benefits in terms of shared experiences [30,51] with others that provide something to talk about (football) as well as an opportunity to talk and connect [16], which is deemed important in recovery $[9,11]$ especially for those involved in medium-secure units who might be, or feel, isolated.

An issue that comes with increased connectedness in this context, which was highlighted previously in a football project [4], was the competitive nature of football and how it could lead to violence in sessions. However, it was noted that participants frequently 
praised these sessions and the 'culture' that meant there was very little conflict, violence or "aggro that we don't wanna see here" (Garth, Service-user). Some suggested the reason for this was the interactive, collective nature of the sessions, as staff and serviceusers played together and were considered, generally, to be equal, or as Jermain (Service-user) put it "on the same level, everyone is the same out on the pitch, no matter where you came from or whether you are staff or usually locked-up". Another factor is the longstanding nature of the sessions, as they have been running, in various forms, for more than a decade, with some participants being involved for that period of time. Therefore, there is a well-established culture or habitus [52] that guides the behaviours, which is especially useful to inculcate new-comers to the sessions.

\section{Empowerment}

The nature of medium-secure units means serviceusers have limited empowerment, but these football sessions demonstrated that this does not always have to be the case. The data supported a number of the subthemes of the empowerment processes that Leamy et al. [9] identified as being important for recovery, with the most recognisable being 'maintaining good physical health and well-being'. It was reported frequently in the interview data that participants were mostly sedentary during the rest of the week, but that these sessions gave them a chance to be active. The general sedentary behaviour of service-users raises questions about other service-users who do not attend these types of sessions, and whether provision (options more appealing to personal tastes than football) should be made more readily available, especially on wards where opportunities and, therefore, choices and empowerment are extremely limited. For Jimmy (Service-user), the opportunity to be active was appreciated:

You'll always see me running, in the game I'm running all the time, non-stop. It's the only chance I get so I get sweating. It's good for my heart, and my weight, 'cause I didn't always look like this. But it's hard, when I'm not here, to run around at all.

Being empowered enough to be able to make a choice [53] is important for 'regaining independence and autonomy' [9]. Participants appreciated how service-users can choose to attend (albeit, if that is an authorised option for them), can be team captains (and choose their teammates), and can choose to attend and not actually play (for instance, there is one service-user who never plays football, but attends almost every week and in the short time gap between games will run a lap of the pitch). This empowerment and taking control of decision making transcends just those experiences of service-users in medium secure units, as participant observation made it possible to witness over time how the sessions provided a safe and familiar space for people as they progressed on their recovery journey [5], a journey that sometimes involved participants who had previously returned to the community becoming more ill and finding themselves back in secure care, but they benefitted from the on-going sessions and the connections they retained, as Greg (Service-user) explained:

I was feeling a lot better a while ago, but I had some troubles again. But you know what, I only missed like four weeks or something [of the football], and they let me keep coming, so that really helped to see the guys. Them people are my friends, it's like coming home. Some of these guys here I've known through the footie for five or six years, we wouldn't have that otherwise.

The football sessions appeared to be a useful tool for the process of regaining independence and autonomy for transitioning service-users who were out of the units or wards, as there remained a support network for them to cohere around whilst they made decisions and recovered their autonomy, for instance, choosing to attend, considering organisation and timing, making transport arrangements, and so on. The final element of the empowerment processes that were evident was how the positive and supportive culture encouraged 'focusing on strengths' [9]. In sessions this included supportive remarks, encouragement 'from the sidelines' from spectators, cheering when someone scored, and generally making people feel good about their footballing ability, which service-users reported contrasts with experiences of some of the language and interactions in clinical settings. This positivity is considered important for recovery processes and making positive changes [3], which shall be discussed in the following sections on hope and identity. 
Hope and Optimism

Previous football and mental wellbeing studies, for instance, Lewis et al. [36] found via quantitative data analysis (often using the Warwick-Edinburgh Mental Wellbeing Scale) that participants felt more optimistic following involvement in such a project. The current study goes beyond this questionnaire and survey data to provide some qualitative elucidation to what this optimism or hope might actually entail, whilst providing a voice for the participants. It was evident that there were elements of being involved in the sessions that encouraged participants to feel more hopeful and optimistic about the future in terms of the short, medium and long-term. In the short term, service-users felt that they really benefitted from having something to look forward to each week, as Ricky (Service-user) outlined: "it's the best bit of my week that's for sure. If I'm honest, like, it's the only good bit of the week mostly, keeps my health going. I'd come every day if they ran it". Ryan (Staff) echoed this from a staff perspective:

These guys look forward to coming, absolutely. I can speak about the patients who I work with, and they talk about it all week, especially if they've had a good performance and scored some good goals. They don't shut up about it [laughter]. They keep telling us how brilliant it is, and it gives them a real focus, and I think that gives us as staff something to work with.

In the medium term, participants appreciated how (perhaps indirectly) the sessions enabled them to see beyond their current circumstances and feel more hopeful about their own health and personal recovery. This was mainly due to the incorporation of serviceusers who had transitioned through the stepped process and had either moved from medium to lowsecure units, or into service accommodation or back into the community, but who still attended the sessions. From spending time with the participants, and seeing these transitions occur, it was possible to see the personal relationships and communication present that gave people hope that things could change. As Jon (Service-user) stated: "It definitely gives me a bit of hope, because I see people come here that aren't even in secure services anymore. People that I know that have got out and they've come back and still chat to me and that, do you know what I mean? It makes you see what you can do, like, and be better". Hardeep (Service-user) also explained: "it gives people hope seeing others that are now back in the community. Some people that have moved on from here, they come back, and I think that's really good, but for them its good, too, so they have somewhere familiar to come, they aren't just on their own out there". There was also the benefit for being optimistic moving forwards about the therapeutic relationships between staff and service-users, as Jasper (Staff) explained:

It's beneficial for everybody involved. Even the members of staff who aren't involved in actually playing can see their patients in a different light, because there are a lot of patients who are stuck on the ward day in, day out and they come here and they're completely different. It's like seeing a completely different person at times.

Almost on a weekly basis, service-user participants expressed what can be interpreted as a longer-term hope of getting well and 'being a footballer' or just joining a local team once they are back in the community, which shall be discussed in relation to identity in the next section.

\section{Identity}

A common theme amongst service-users was how they had played football a great deal prior to becoming ill. During the participant observation data collection there were very often discussions about the teams they had played for or the level they had reached, and it was frequently followed by a reflection of how pleased they were that these sessions were available to them to 'recover' that 'old' part of their identity, whilst providing hope that this could be enhanced further in the future. In addition to being an important element of their perception of self [16] and giving life value and meaning [54], there is potentially a benefit to their social identity in that playing football again affords them cultural capital [55], which is valued in this different 'field' [52] (i.e., in a football arena in the town centre) that contrasts so markedly from their usual social environment (being in a unit or on a ward) that does not value such capital, and therefore can make that person feel undervalued. Furthermore, service-users, volunteers and staff appreciated the 
dynamic of everyone playing together, as Sean (Service-user) stated:

The power dynamics aren't that obvious, everyone's on one level. I can't praise it enough. It's good that here I'm better than the staff [at football] and we have more of a laugh about that, whereas the rest of the time I'm just 'Sean' the patient.

This is in line with the more collective, solidarityenhancing activities that have been called for by Leamy et al. [9] and McKeown et al. [30] which highlighted the benefits of flexible inter-personal relations in settings that contrast with the 'mainstream' mental health service settings and relations with practitioners. This significance is perhaps intensified in medium-secure units where these relations and power dynamics are especially manifest, as Onken et al. (53, p10) suggested the "interaction among characteristics of the individual (such as hope), characteristics of the environment (such as opportunities), and characteristics of the exchange between the individual and the environment (such as choice), can promote or hinder recovery", therefore services could benefit from reflecting on service-users' hope and identity when considering opportunities and choices.

\section{Meaningful}

Although the data suggested that this element of the recovery processes framework was discussed the least by participants, there was a strong consensus of football being meaningful and the sessions meaning a lot to them in terms of their health and ongoing recovery. Participants felt particularly strongly when asked 'what if the sessions stopped?'. Put simply by Megan (Service-user): "If the sessions weren't on I think I might fall back into depression. A lot of people would be lost without this, I think. I know I would". These sentiments were echoed by staff members Lewis and Mikey: "for some of the guys who come, it's the only physical exercise they do. It's the only social thing they do" (Lewis, Staff); "I dread to think, mate [what would happen if the sessions stopped]. I dread to think. They say to me 'What would I do on my own on a Tuesday?', they all love football and want to come here" (Mikey, Staff). A functionalist perspective [56] would highlight the function of sport of being the 'hook' that brings people together, in order for additional benefits (such as the other CHIME recovery processes) to be enabled. The obvious limitation in a practical sense for services is that not everyone likes football and facilities might not be available, however other sports could be offered and despite the limited funding there is a growing body of evidence that is highlighting how sport can really influence people's personal recovery, so these opportunities arguably should be made available.

\section{Academic and Practical Impact}

In an academic sense, this study has attempted to add to the limited, but growing, evidence base in this area in four specific ways, by exploring sport in a specific mental health context (that has not previously been studied); whilst focusing on the lived experiences of those involved; via an ethnographic approach; the analysis of which is underpinned by the CHIME conceptual framework [9]. In terms of mental health practice and impact, the findings and report produced for the Trust that runs the sessions has led to a documented increased awareness (especially at a senior management level) of the benefits of the sessions for staff and service-users, as well as a formally reported appreciation of the benefits for recovery from taking part in sport. This has subsequently led to funding being secured for future football sessions (that was not previously forthcoming) and has also contributed to a strategy being implemented to increase sport and physical activity across the Trust, so more people are benefitting from the sessions on a continuing basis. Considerations outside of the CHIME analysis here could adopt a critical approach and point to elements such as the gender divide during sessions [46], competitive sport causing conflict [4] or the predominance of the biomedical model that still underpins services [2]. However, this study explored the lived experiences of those involved in the sessions, and these experiences were overwhelmingly positive. Even when probed, the only negative comments related to frustrations that funding was precarious (which caused anxiety about sessions not continuing), wanting to have more sessions during the week available and wanting to play for longer during sessions. The data demonstrated that these sessions have considerable links to the CHIME processes, and 
can therefore be considered to contribute to personal recovery for those involved. Therefore, this study has responded to the challenge of Leamy et al. $(9, \mathrm{p} 451)$ to use the CHIME framework to develop an evidence base that "simultaneously helps mental health professionals to support recovery and respects the understanding that recovery is a unique and individual experience rather than something the mental health system does to a person".

Acknowledgements Special thanks to Robert Hipkiss and Jane Clark, and all of the service-users, volunteers and staff involved in the football sessions.

Open Access This article is distributed under the terms of the Creative Commons Attribution 4.0 International License (http:// creativecommons.org/licenses/by/4.0/), which permits unrestricted use, distribution, and reproduction in any medium, provided you give appropriate credit to the original author(s) and the source, provide a link to the Creative Commons license, and indicate if changes were made.

\section{References}

1. Ramon S, Healy B, Renouf N. Recovery from mental illness as an emergent concept and practice in Australia and the UK. Int J Soc Psychiatry. 2007;53(2):108-22.

2. Watson DP. The evolving understanding of recovery: what does the sociology of mental health have to offer? Hum Soc. 2012;36(4):290-308.

3. Slade M, Longden E. Empirical evidence about recovery and mental health. BMC Psychiatry. 2015;15(1):285.

4. Magee J, Spaaij R, Jeanes R. "It's Recovery United for Me": promises and pitfalls of football as part of mental health recovery. Sociol Sport J. 2015;32(4):357-76.

5. Markowitz FE, Angell B, Greenberg JS. Stigma, reflected appraisals, and recovery outcomes in mental illness. Soc Psychol Q. 2011;74(2):144-65.

6. Borg M, Davidson L. The nature of recovery as lived in everyday experience. J Ment Health. 2008;17(2):129-40.

7. Slade M. Personal recovery and mental illness: a guide for mental health professionals. Cambridge: Cambridge University Press; 2009.

8. Perkins R, Slade M. Recovery in England: transforming statutory services? Int Rev Psychiatry. 2009;24(1):29-39.

9. Leamy M, Bird V, Le Boutillier C, Williams J, Slade M. Conceptual framework for personal recovery in mental health: systematic review and narrative synthesis. $\mathrm{Br} \mathrm{J}$ Psychiatry. 2011;199(6):445-52.

10. Sterling EW, Silke A, Tucker S, Fricks L, Druss BG. Integrating wellness, recovery, and self-management for mental health consumers. Community Ment Health J. 2010;46(2):130-8.

11. Tew J, Ramon S, Slade M, Bird V, Melton J, Le Boutillier C. Social factors and recovery from mental health difficulties: a review of the evidence. $\mathrm{Br} \mathrm{J}$ Soc Work. 2012;42(3):443-60.

12. Sweeney A, Davies J, McLaren S, Whittock M, Lemma F, Belling R, Clement S, Burns T, Catty J, Jones IR, Rose D. Defining continuity of care from the perspectives of mental health service users and professionals: an exploratory, comparative study. Health Expect. 2016;19(4):973-87.

13. Schrank B, Brownell T, Riches S, Chevalier A, Jakaite Z, Larkin C, Lawrence V, Slade M. Staff views on wellbeing for themselves and for service users. J Ment Health. 2015;24(1):48-53.

14. Morera T, Bucci S, Randal C, Barrett M, Pratt D. Exploring views about mindfulness groups for voice-hearing from the perspective of service users and staff: a Q-methodology study. Psychother Res. 2017;27(2):179-88.

15. Rose D, Evans J, Laker C, Wykes T. Life in acute mental health settings: experiences and perceptions of service users and nurses. Epidemiol Psychiatr Sci. 2015;24(1):90-6.

16. Carless D, Douglas K. The role of sport and exercise in recovery from serious mental illness: two case studies. Int $\mathbf{J}$ Mens Health. 2008;7(2):137-56.

17. Brawn P, Combes H, Ellis N. Football narratives: recovery and mental health. J New Writ Health Soc Care. 2015;1(2):30-46.

18. Rosenbaum S, Tiedemann A, Ward PB. Meta-analysis physical activity interventions for people with mental illness: a systematic review and meta-analysis. J Clin Psychiatry. 2014;75:964-74.

19. Rosenbaum S, Vancampfort D, Steel Z, Newby J, Ward PB, Stubbs B. Physical activity in the treatment of post-traumatic stress disorder: a systematic review and meta-analysis. Psychiatry Res. 2015;230(2):130-6.

20. Foucault M. Discipline and Punish, trans. Alan Sheridan (New York: Vintage, 1979). 1977;191.

21. Craft LL. Exercise and clinical depression: examining two psychological mechanisms. Psychol Sport Exerc. 2005;6(2):151-71.

22. Sylvester BD, Mack DE, Busseri MA, Wilson PM, Beauchamp MR. Health-enhancing physical activity, psychological needs satisfaction, and well-being: is it how often, how long, or how much effort that matters? Ment Health Phys Act. 2012;5(2):141-7.

23. Pareja-Galeano H, Mayero S, Perales M, Garatachea N, Santos-Lozano A, Fiuza-Luces C, Emanuele E, Gálvez G, Sanchis-Gomar B, Lucia A. Biological rationale for regular physical exercise as an effective intervention for the prevention and treatment of depressive disorders. Curr Pharm Des. 2016;22(24):3764-75.

24. Department of Health. Future in mind: promoting, protecting and improving our children and young people's mental health and wellbeing. London: Department of Health; 2015.

25. Smith A, Jones J, Houghton L, Duffell T. A political spectator sport or policy priority? A review of sport, physical activity and public mental health policy. Int J Sport Policy Polit. 2016;8(4):593-607.

26. HM Government. Sporting future: a new strategy for an active nation. London: Cabinet Office; 2015.

27. Henderson C, O'Hara S, Thornicroft G, Webber M. Corporate social responsibility and mental health: the Premier League football Imagine Your Goals programme. Int Rev Psychiatry. 2014;26(4):460-6. 
28. Pringle A. The growing role of football as a vehicle for interventions in mental health care. J Psychiatr Ment Health Nurs. 2009;16(6):553-7.

29. Jones A. Football as a metaphor: learning to cope with life, manage emotional illness and maintain health through to recovery. J Psychiatr Ment Health Nurs. 2009;16(5):488-92.

30. McKeown M, Roy A, Spandler H. 'You'll never walk alone': supportive social relations in a football and mental health project. Int J Ment Health Nurs. 2015;24(4):360-9.

31. Spandler H, Mckeown M, Roy A, Hurley M. Football metaphor and mental well-being: an evaluation of the It's a Goal! programme. J Ment Health. 2013;22(6):544-54.

32. Mason OJ, Holt R. A role for football in mental health: the Coping Through Football project. Psychiatrist. 2012;36(8):290-3.

33. Spandler H, Roy A, Mckeown M. Using football metaphor to engage men in therapeutic support. J Soc Work Pract. 2014;28(2):229-45.

34. Doherty DT, Kartalova-O'Doherty Y. Gender and self-reported mental health problems: predictors of help seeking from a general practitioner. $\mathrm{Br} \mathrm{J}$ Health Psychol. 2010;15(1):213-28.

35. Spandler H, McKeown M. A critical exploration of using football in health and welfare programs: gender, masculinities, and social relations. J Sport Soc Issues. 2012;36(4):387-409.

36. Lewis CJ, Reeves MJ, Roberts SJ. Improving the physical and mental well-being of typically hard-to-reach men: an investigation of the impact of the Active Rovers project. Sport Soc. 2017;20(2):258-68.

37. Pringle A, Sayers P. It's a Goal! The half-time score. Ment Health Nurs. 2006;26(3):14-7.

38. Klein AM. Little big men: bodybuilding subculture and gender construction. Albany: Suny Press; 1993.

39. Smith B, Sparkes AC. Qualitative research methods in sport, exercise and health: from process to product. Abingdon: Routledge; 2014.

40. Williams TL, Smith B, Papathomas A. Physical activity promotion for people with spinal cord injury: physiotherapists' beliefs and actions. Disabil Rehabil. 2018;40(1):52-61.

41. Beebe LH, Tian L, Morris N, Goodwin A, Allen SS, Kuldau J. Effects of exercise on mental and physical health parameters of persons with schizophrenia. Issues Ment Health Nurs. 2005;26(6):661-76.
42. Horsfall J, Cleary M, Hunt GE. Stigma in mental health: clients and professionals. Issues Ment Health Nurs. 2010;31(7):450-5.

43. Mason OJ, Holt R. Mental health and physical activity interventions: a review of the qualitative literature. J Ment Health. 2012;21(3):274-84.

44. Gold RL. Roles in sociological field observations. Soc Forces. 1958;36:217.

45. Jones I, Brown L, Holloway I. Qualitative research in sport and physical activity. Thousand Oaks: Sage; 2013.

46. Spandler H, Roy A, McKeown M. Playing by the rules? Gender relations in a football and mental health project. J Mens Stud. 2014;22(2):140-54.

47. Carroll P, Kirwan L, Lambe B. Engaging 'hard to reach' men in community based health promotions. Int $\mathbf{J}$ Health Promot Educ. 2014;52(3):120-30.

48. White A, Witty K. Men's under use of health servicesfinding alternative approaches. J Ment Health. 2009;6(2):95-7.

49. Silk M. Sporting ethnography: philosophy, methodology and reflection. In: Mason D, Andrews D, Silk ML, editors. Qualitative methods for sports studies. Oxford: Berg; 2005. p. 65-103.

50. Brijnath B. Applying the CHIME recovery framework in two culturally diverse Australian communities: qualitative results. Int J Soc Psychiatry. 2015;61(7):660-7.

51. Fogarty M, Happell B. Exploring the benefits of an exercise program for people with schizophrenia: a qualitative study. Issues Ment Health Nurs. 2005;26(3):341-51.

52. Bourdieu P. Distinction: a social critique of the judgement of taste. Abingdon: Routledge; 1984.

53. Onken SJ, Craig CM, Ridgway P, Ralph RO, Cook JA. An analysis of the definitions and elements of recovery: a review of the literature. Psychiatr Rehabil J. 2007;31(1):9-22.

54. Repper J, Perkins R. Social inclusion and recovery: a model for mental health practice. Amsterdam: Elsevier Health Sciences; 2003.

55. Bourdieu P. Sport and social class. Information (International Social Science Council). 1978;17(6):819-40.

56. Giulianotti R. Sport: a critical sociology. Hoboken: Wiley; 2016.

Publisher's Note Springer Nature remains neutral with regard to jurisdictional claims in published maps and institutional affiliations. 